UFIFT-QG-10-06

\title{
Solving the Effective Field Equations for the Newtonian Potential
}

\author{
Sohyun Park ${ }^{\dagger}$ and R. P. Woodard ${ }^{\ddagger}$ \\ Department of Physics \\ University of Florida \\ Gainesville, FL 32611
}

\begin{abstract}
Loop corrections to the gravitational potential are usually inferred from scattering amplitudes, which seems quite different from how the linearized Einstein equations are solved with a static, point mass to give the classical potential. In this study we show how the Schwinger-Keldysh effective field equations can be used to compute loop corrections to the potential in a way which parallels the classical treatment. We derive explicit results for the one loop correction from the graviton self-energy induced by a massless, minimally coupled scalar.
\end{abstract}

PACS numbers: 04.20.Cv, 02.40.Ky, 04.60.-m, 98.80.-k

† e-mail: spark@phys.ufl.edu

$\ddagger$ e-mail: woodard@phys.ufl.edu 


\section{Introduction}

Everyone who has studied general relativity is familiar with solving the linearized Einstein equations to derive the first correction due to a point particle. One expands the full metric about flat space,

$$
d s^{2} \equiv\left[\eta_{\mu \nu}+h_{\mu \nu}(t, \vec{x})\right] d x^{\mu} d x^{\nu}=-c^{2} d t^{2}+d \vec{x} \cdot d \vec{x}+h_{\mu \nu}(t, \vec{x}) d x^{\mu} d x^{\nu} .
$$

Linearizing the full Einstein equation gives,

$$
R^{\mu \nu}-\frac{1}{2} g^{\mu \nu} R=\frac{8 \pi G}{c^{2}} \times T^{\mu \nu} \Longrightarrow \mathcal{D}^{\mu \nu \rho \sigma} h_{\rho \sigma}(t, \vec{x})=\frac{8 \pi G}{c^{2}} \times M \delta_{0}^{\mu} \delta_{0}^{\nu} \delta^{3}(\vec{x}),
$$

where the flat space Lichnerowicz operator is,

$$
\mathcal{D}^{\mu \nu \rho \sigma} \equiv \frac{1}{2}\left[\eta^{\mu \nu} \eta^{\rho \sigma} \partial^{2}-\partial^{\mu} \partial^{\nu} \eta^{\rho \sigma}-\eta^{\mu \nu} \partial^{\rho} \partial^{\sigma}-\eta^{\mu(\rho} \eta^{\sigma) \nu} \partial^{2}+2 \partial^{(\mu} \eta^{\nu)(\rho} \partial^{\sigma)}\right] .
$$

Up to a linearized gauge transformation, the solution is,

$$
h_{00}=\frac{2 G M}{c^{2} r} \quad, \quad h_{0 i}=h_{i 0}=0 \quad, \quad h_{i j}=\frac{2 G M}{c^{2} r} \widehat{r}^{i} \widehat{r}^{j}
$$

where $r \equiv|\vec{x}|$ and $\widehat{r}^{i} \equiv x^{i} / r$.

This is simple to understand, and easy to extend to more complicated sources which can even change with time. Yet we are told that a completely different technique must be employed in order to include quantum corrections. Instead of solving the effective field equations, one computes the gravitational contribution to the amplitude for 2-particle scattering. Then the inverse scattering problem is used to reconstruct the potentials. For matter particles one only needs to correct the graviton propagator, which was done long ago for vectors [1, 2], spin $\frac{1}{2}$ fermions [3] and scalars [4]. The general result for the long range Newtonian potential is [5, 6],

$$
h_{00} \longrightarrow \frac{2 G M}{c^{2} r}\left\{1+\left[N_{0}+3 N_{\frac{1}{2}}+12 N_{1}\right] \frac{\hbar G}{45 \pi c^{3} r^{2}}+O\left(G^{2}\right)\right\},
$$

where $N_{s}$ indicates the number of particles of spin $s$ contributing to the graviton self-energy, and it should be noted that the $s=0$ result is for a massless, conformally coupled scalar. (The correction for a massless, minimally cou-

pled scalar does not seem to have been published.) Including gravitons is 
very much harder and has been the work of decades because one must also correct the vertices [1, 7, [8, 9, 10, 11]. The generally accepted result seems to be [12],

$$
h_{00} \longrightarrow \frac{2 G M}{c^{2} r}\left\{1+\frac{41 \hbar G}{10 \pi c^{3} r^{2}}+O\left(G^{2}\right)\right\},
$$

The scattering amplitude technique is certainly correct, although there have been many disputes about its application to graviton corrections [11]. What bewilders is the contrast between it and the classical procedure. Why must we do an asymptotic scattering experiment to infer the gravitational force in quantum field theory when we can observe orbits over finite times to get it classically? And what would we do for a dynamic source which was evolving? The notion of quantum-corrected, effective field equations is more than half a century old [13]; why is there not some way of solving them in analogy to the classical computation?

In fact there is such a technique, and we will employ it here to solve for the one loop change in the gravitational potentials due to a massless, minimally coupled scalar field. The results for other massless particles have long been known for flat space [5, 6], and the method we describe can be used as well in de Sitter background, for which the absence of an S-matrix [14, 15] precludes the scattering amplitude technique. This paper will serve as a warm-up exercise for that more challenging computation, as well as an important correspondence limit.

Section 2 is devoted to explaining the general formalism. The actual

computation is done in section 3. Our results are summarized and discussed in section 4.

\section{Schwinger-Keldysh Effective Field Eqns}

The graviton self-energy $-i\left[{ }^{\mu \nu} \Sigma^{\rho \sigma}\right]\left(x ; x^{\prime}\right)$ is the one-particle-irreducible (1PI) 2 -point function for the graviton field $h_{\mu \nu}(t, \vec{x})$. It serves to quantum correct the linearized Einstein equation (2),

$$
\mathcal{D}^{\mu \nu \rho \sigma} h_{\rho \sigma}(x)+\int d^{4} x^{\prime}\left[\mu \nu \Sigma^{\rho \sigma}\right]\left(x ; x^{\prime}\right) h_{\rho \sigma}\left(x^{\prime}\right)=\frac{8 \pi G}{c^{2}} M \delta_{0}^{\mu} \delta_{0}^{\nu} \delta^{3}(\vec{x}) .
$$

However, this equation suffers from two embarrassments:

- It isn't causal because the in-out self-energy is nonzero for points $x^{\prime \mu}$ which are spacelike separated from $x^{\mu}$, or lie to its future; and 
- It doesn't produce real potentials $h_{\mu \nu}$ because the in-out self-energy has an imaginary part.

One can get the right result for a static potential by simply ignoring the imaginary part [5, 6, 8], but circumventing the limitations of the in-out formalism becomes more and more difficult as time dependent sources and higher order corrections are included, and these techniques break down entirely for the case of cosmology in which there may not even be asymptotic vacua. It is not that the in-out self-energy is somehow "wrong". In fact, it is exactly the right thing to correct the Feynman propagator for asymptotic scattering computations in flat space. The point is rather that equation (17) doesn't provide the generalization we seek of the classical field equation (2).

The better technique is known as the Schwinger-Keldysh formalism [16]. It provides a way of computing true expectation values that is almost as simple as the Feynman diagrams which produce in-out matrix elements. The Schwinger-Keldysh rules are best stated in the context of a scalar field $\varphi(x)$ whose Lagrangian (the space integral of its Lagrangian density) at time $t$ is $L[\varphi(t)]$. Suppose we are given a Heisenberg state $|\Psi\rangle$ whose wave functional in terms of the operator eigenkets at time $t_{0}$ is $\Psi\left[\varphi\left(t_{0}\right)\right]$, and we wish to take the expectation value, in the presence of this state, of a product of two functionals of the field operator: $A[\varphi]$, which is anti-time-ordered, and $B[\varphi]$, which is time-ordered. The Schwinger-Keldysh functional integral for this is [17],

$$
\begin{aligned}
\langle\Psi|A[\varphi] B[\varphi]| \Psi\rangle & =\left[\left[d \varphi_{+}\right]\left[d \varphi_{-}\right] \delta\left[\varphi_{-}\left(t_{1}\right)-\varphi_{+}\left(t_{1}\right)\right]\right. \\
& \left.\times A\left[\varphi_{-}\right] B\left[\varphi_{+}\right] \Psi^{*}\left[\varphi_{-}\left(t_{0}\right)\right] e^{i \int_{t_{0}}^{t_{1}} d t\left\{L\left[\varphi_{+}(t)\right]-L\left[\varphi_{-}(t)\right]\right.}\right\} \Psi\left[\varphi_{+}\left(t_{0}\right)\right] .
\end{aligned}
$$

The time $t_{1}>t_{0}$ is arbitrary as long as it is later than the latest operator which is contained in either $A[\varphi]$ or $B[\varphi]$.

The Schwinger-Keldysh rules can be read off from its functional representation (8). Because the same field operator is represented by two different dummy functional variables, $\varphi_{ \pm}(x)$, the endpoints of lines carry a \pm polarity. External lines associated with the anti-time-ordered operator $A[\varphi]$ have the polarity whereas those associated with the time-ordered operator $B[\varphi]$ have the + polarity. Interaction vertices are either all + or all - . Vertices with + polarity are the same as in the usual Feynman rules whereas vertices with the - polarity have an additional minus sign. If the state $|\Psi\rangle$ is something 
other than free vacuum then it contributes additional interaction vertices on the initial value surface [18].

Propagators can be,,+++--+ , or -- . All four polarity variations can be read off from the fundamental relation (8) when the free Lagrangian is substituted for the full one. It is useful to denote canonical expectation values in the free theory with a subscript 0 . With this convention we see that the ++ propagator is just the ordinary Feynman propagator,

$$
i \Delta_{++}\left(x ; x^{\prime}\right)=\left\langle\Omega\left|T\left(\varphi(x) \varphi\left(x^{\prime}\right)\right)\right| \Omega\right\rangle_{0}=i \Delta\left(x ; x^{\prime}\right),
$$

where $T$ stands for time-ordering and $\bar{T}$ denotes anti-time-ordering. The other polarity variations are simple to read off and to relate to the Feynman propagator,

$$
\begin{aligned}
& i \Delta_{-+}\left(x ; x^{\prime}\right)=\left\langle\Omega\left|\varphi(x) \varphi\left(x^{\prime}\right)\right| \Omega\right\rangle_{0}=\theta\left(t-t^{\prime}\right) i \Delta\left(x ; x^{\prime}\right)+\theta\left(t^{\prime}-t\right)\left[i \Delta\left(x ; x^{\prime}\right)\right]^{*}, \\
& i \Delta_{+-}\left(x ; x^{\prime}\right)=\left\langle\Omega\left|\varphi\left(x^{\prime}\right) \varphi(x)\right| \Omega\right\rangle_{0}=\theta\left(t-t^{\prime}\right)\left[i \Delta\left(x ; x^{\prime}\right)\right]^{*}+\theta\left(t^{\prime}-t\right) i \Delta\left(x ; x^{\prime}\right), \\
& i \Delta_{--}\left(x ; x^{\prime}\right)=\left\langle\Omega\left|\bar{T}\left(\varphi(x) \varphi\left(x^{\prime}\right)\right)\right| \Omega\right\rangle_{0}=\left[i \Delta\left(x ; x^{\prime}\right)\right]^{*}
\end{aligned}
$$

Therefore we can get the four propagators of the Schwinger-Keldysh formalism from the Feynman propagator once that is known.

Because external lines can be either + or - in the Schwinger-Keldysh formalism, every 1PI N-point function of the in-out formalism gives rise to $2^{N}$ 1PI N-point functions in the Schwinger-Keldysh formalism. For every classical field $\phi(x)$ of an in-out effective action, the coorresponding SchwingerKeldysh effective action must depend upon two fields - call them $\phi_{+}(x)$ and $\phi_{-}(x)$ - in order to access the appropriate 1PI function [19]. For the scalar paradigm we have been considering this effective action takes the form,

$$
\begin{aligned}
& \Gamma\left[\phi_{+}, \phi_{-}\right]=S\left[\phi_{+}\right]-S\left[\phi_{-}\right]-\frac{1}{2} \int d^{4} x \int d^{4} x^{\prime} \\
& \quad \times\left\{\begin{array}{c}
\phi_{+}(x) M_{++}^{2}\left(x ; x^{\prime}\right) \phi_{+}\left(x^{\prime}\right)+\phi_{+}(x) M_{++}^{2}\left(x ; x^{\prime}\right) \phi_{-}\left(x^{\prime}\right) \\
+\phi_{-}(x) M_{-+}^{2}\left(x ; x^{\prime}\right) \phi_{+}\left(x^{\prime}\right)+\phi_{-}(x) M_{--}^{2}\left(x ; x^{\prime}\right) \phi_{-}\left(x^{\prime}\right)
\end{array}\right\}+O\left(\phi_{ \pm}^{3}\right),
\end{aligned}
$$

where $S$ is the classical action. The effective field equations are obtained by varying with respect to $\phi_{+}$and then setting both fields equal [19],

$$
\left.\frac{\delta \Gamma\left[\phi_{+}, \phi_{-}\right]}{\delta \phi_{+}(x)}\right|_{\phi_{ \pm}=\phi}=\left[\partial^{2}-m^{2}\right] \phi(x)-\int d^{4} x^{\prime}\left[M_{++}^{2}\left(x ; x^{\prime}\right)+M_{+-}^{2}\left(x ; x^{\prime}\right)\right] \phi\left(x^{\prime}\right)+O\left(\phi^{2}\right) \text {. }
$$


The two 1PI 2-point functions we would need to quantum correct the linearized scalar field equation are $M_{++}^{2}\left(x ; x^{\prime}\right)$ and $M_{+-}^{2}\left(x ; x^{\prime}\right)$. Their sum in (14) gives effective field equations which are causal in the sense that the two 1PI functions cancel unless $x^{\prime \mu}$ lies on or within the past light-cone of $x^{\mu}$. Their sum is also real, which neither 1PI function is separately.

As mentioned before, the point of the present paper is to lay the groundwork for a computation of the one loop correction to the force of gravity on de Sitter background. The graviton contribution to the self-energy was computed some years ago [20] but has never been used in the effective field equations. A computation of the scalar contribution is underway.

Although the current computation will be the first to explore corrections to a force law, the linearized effective field equations have been studied on de Sitter background for many simpler models. In scalar quantum electrodynamics the one loop vacuum polarization was computed and used to correct for the propagation of dynamical photons [21, 22], but not yet for the Coulomb force. The one loop scalar self-mass-squared has also been used to correct for the propagation of charged, massless, minimally coupled scalars [23]. Both the fermion [24] and scalar [25] 1PI 2-point functions of Yukawa theory have been computed and used to correct the mode functions. The one and two loop scalar self-mass-squared of $\lambda \varphi^{4}$ theory has been computed and used to correct for the propagation of massless, minimally coupled scalars [26]. In Einstein + Dirac the one loop fermion self-energy has been computed and used to correct the fermion mode function [27]. And the same thing has been done for scalars in Scalar + Einstein [28].

\section{Solving for the Potentials}

This section is the heart of the paper. We begin by expressing the linearized effective field equations in a form which is both manifestly real and causal. We then explain how these equations can be solved perturbatively. The hardest step is integrating the one loop self-energy against the tree order solution. The section closes by working out the two one loop potentials.

\subsection{Achieving A Manifestly Real and Causal Form}

The basis for our work is a position space result for the one loop contribution to the 1PI graviton 2-point function from a loop of massless, minimally 
coupled scalars, using dimensional regularization and a minimal choice for the higher derivative counterterms [17]. (Previous Schwinger-Keldysh computations of this quantity had been given in momentum space [29] which is not as useful for us.) All four polarization variations take the form,

$$
\left[{ }^{\mu \nu} \Sigma_{ \pm \pm}^{\rho \sigma}\right]\left(x ; x^{\prime}\right)=\mathrm{D}^{\mu \nu \rho \sigma} \Sigma_{ \pm \pm}\left(x ; x^{\prime}\right)
$$

where the 4th-order, tensor-differential operator is,

$\mathrm{D}^{\mu \nu \rho \sigma} \equiv\left[\eta^{\mu \nu} \partial^{2}-\partial^{\mu} \partial^{\nu}\right]\left[\eta^{\rho \sigma} \partial^{2}-\partial^{\rho} \partial^{\sigma}\right]+\frac{1}{3}\left[\eta^{\mu(\rho} \eta^{\sigma) \nu} \partial^{4}-2 \partial^{(\mu} \eta^{\nu)(\rho} \partial^{\sigma)}+\partial^{\mu} \partial^{\nu} \partial^{\sigma} \partial^{\rho}\right]$.

The four bi-scalars are,

$$
\Sigma_{ \pm \pm}\left(x ; x^{\prime}\right)=( \pm)( \pm) \frac{i \kappa^{2} \partial^{2}}{5120 \pi^{4}}\left[\frac{\ln \left(\mu^{2} \Delta x_{ \pm \pm}^{2}\right)}{\Delta x_{ \pm \pm}^{2}}\right]
$$

where $\mu^{2}$ is the usual scale of dimensional regularization, $\kappa^{2} \equiv 16 \pi \hbar G / c^{3}$ is the loop-counting parameter of quantum gravity and the four SchwingerKeldysh length functions are,

$$
\begin{aligned}
\Delta x_{++}^{2}\left(x ; x^{\prime}\right) & \equiv\left\|\vec{x}-\vec{x}^{\prime}\right\|^{2}-c^{2}\left(\left|t-t^{\prime}\right|-i \epsilon\right)^{2}, \\
\Delta x_{+-}^{2}\left(x ; x^{\prime}\right) & \equiv\left\|\vec{x}-\vec{x}^{\prime}\right\|^{2}-c^{2}\left(t-t^{\prime}+i \epsilon\right)^{2}, \\
\Delta x_{-+}^{2}\left(x ; x^{\prime}\right) & \equiv\left\|\vec{x}-\vec{x}^{\prime}\right\|^{2}-c^{2}\left(t-t^{\prime}-i \epsilon\right)^{2}, \\
\Delta x_{--}^{2}\left(x ; x^{\prime}\right) & \equiv\left\|\vec{x}-\vec{x}^{\prime}\right\|^{2}-c^{2}\left(\left|t-t^{\prime}\right|+i \epsilon\right)^{2} .
\end{aligned}
$$

Although the divergent parts of (15) have been subtracted off [17], it should be noted that they agree exactly with those originally found by 't Hooft and Veltman [30].

We can achieve a significant simplification by first extracting another d'Alembertian from (17),

$$
\Sigma_{ \pm \pm}\left(x ; x^{\prime}\right)=( \pm)( \pm) \frac{i \kappa^{2} \partial^{4}}{40960 \pi^{4}}\left[\ln ^{2}\left(\mu^{2} \Delta x_{ \pm \pm}^{2}\right)-2 \ln \left(\mu^{2} \Delta x_{ \pm \pm}^{2}\right)\right] .
$$

Now define the position and temporal separations, and the associated invariant length-squared,

$$
\Delta r \equiv\left\|\vec{x}-\vec{x}^{\prime}\right\| \quad, \quad \Delta t \equiv t-t^{\prime} \quad, \quad \Delta x^{2} \equiv \Delta r^{2}-c^{2} \Delta t^{2} .
$$


The ++ and +- logarithms can be expanded in terms of their real and imaginary parts,

$$
\begin{aligned}
& \ln \left(\mu^{2} \Delta x_{++}^{2}\right)=\ln \left(\mu^{2}\left|\Delta x^{2}\right|\right)+i \pi \theta\left(-\Delta x^{2}\right), \\
& \ln \left(\mu^{2} \Delta x_{+-}^{2}\right)=\ln \left(\mu^{2}\left|\Delta x^{2}\right|\right)-i \pi \operatorname{sgn}(\Delta t) \theta\left(-\Delta x^{2}\right) .
\end{aligned}
$$

The ++ and +- logarithms agree for spacelike separation $\left(\Delta x^{2}>0\right)$, and for $t^{\prime}>t$, whereas they are complex conjugates of one another for $x^{\prime \mu}=\left(c t^{\prime}, \vec{x}^{\prime}\right)$ in the past light-cone of $x^{\mu}=(c t, \vec{x})$. Hence the sum of $\Sigma_{++}\left(x ; x^{\prime}\right)$ and $\Sigma_{+-}\left(x ; x^{\prime}\right)$ is both causal and real,

$$
\Sigma_{++}\left(x ; x^{\prime}\right)+\Sigma_{+-}\left(x ; x^{\prime}\right)=-\frac{\kappa^{2} \partial^{4}}{10240 \pi^{3}} \theta(c \Delta t-\Delta r)\left[\ln \left(-\mu^{2} \Delta x^{2}\right)-1\right] .
$$

Let us assume that the state is released in free vacuum at time $t=0$. Our final result for the linearized, one loop effective field equations is,

$$
\begin{aligned}
\mathcal{D}^{\mu \nu \rho \sigma} h_{\rho \sigma}(t, \vec{x})-\frac{\kappa^{2} \mathrm{D}^{\mu \nu \rho \sigma} \partial^{4}}{10240 \pi^{3}} \int_{0}^{t} d c t^{\prime} \int d^{3} x^{\prime} \theta(c \Delta t-\Delta r) \\
\times\left[\ln \left(-\mu^{2} \Delta x^{2}\right)-1\right] h_{\rho \sigma}\left(t^{\prime}, \vec{x}^{\prime}\right)=\frac{8 \pi G M}{c^{2}} \delta_{0}^{\mu} \delta_{0}^{\nu} \delta^{3}(\vec{x}) .
\end{aligned}
$$

Recall that $\kappa^{2} \equiv 16 \pi \hbar G / c^{3}$ is the loop counting parameter of quantum gravity, the Lichnerowitz operator $\mathcal{D}^{\mu \nu \rho \sigma}$ was given in (3) and the 4th order differential operator $\mathrm{D}^{\mu \nu \rho \sigma}$ was given in (16).

\subsection{Solving the Equation Perturbatively}

There is no point in trying to solve equation (27) exactly because it only includes the one loop graviton self-energy. A better approach is to seek a perturbative solution in powers of the loop counting parameter $\kappa^{2}$,

$$
h_{\mu \nu}(t, \vec{x})=\sum_{\ell=0}^{\infty} \kappa^{2 \ell} h_{\mu \nu}^{(\ell)}(t, \vec{x}) .
$$

Of course the $\ell=0$ term obeys the linearized Einstein equation whose solution in Schwarzschild coordinates is,

$$
\mathcal{D}^{\mu \nu \rho \sigma} h_{\rho \sigma}^{(0)}(t, \vec{x})=\frac{8 \pi G M}{c^{2}} \delta_{0}^{\mu} \delta_{0}^{\nu} \delta^{3}(\vec{x}) \Longrightarrow h_{00}^{(0)}=\frac{2 G M}{c^{2} r}, h_{i j}^{(0)}=\frac{2 G M}{c^{2} r} \widehat{r}^{i} \widehat{r}^{j} .
$$


The one loop correction $h_{\mu \nu}^{(1)}$ obeys the equation,

$$
\mathcal{D}^{\mu \nu \rho \sigma} h_{\rho \sigma}^{(1)}(t, \vec{x})=\frac{\mathrm{D}^{\mu \nu \rho \sigma} \partial^{4}}{10240 \pi^{3}} \int_{0}^{t} d t^{\prime} \int d^{3} x^{\prime} \theta(\Delta t-\Delta r)\left[\ln \left(-\mu^{2} \Delta x^{2}\right)-1\right] h_{\rho \sigma}^{(0)}\left(t^{\prime}, \vec{x}^{\prime}\right) .
$$

Finding the two loop correction $h_{\mu \nu}^{(2)}$ would require the two loop self-energy, which we do not have, so $h_{\mu \nu}^{(1)}$ is as high as we can go.

\subsection{The One Loop Source Term}

In this subsection we evaluate the right hand side of equation(30), which sources the one loop correction $h_{\mu \nu}^{(1)}(t, \vec{x})$. This is done in three steps: we first perform the integral, then act the $\partial^{4}$, and finally act the $\mathrm{D}^{\mu \nu \rho \sigma}$.

From the form of the tree order potentials (29) it is apparent that we need two integrals. The first comes from $h_{00}^{(0)}$,

$$
\int_{0}^{t} d t^{\prime} \int d^{3} x^{\prime} \theta(\Delta t-\Delta r)\left[\ln \left(-\mu^{2} \Delta x^{2}\right)-1\right] \times \frac{1}{\left\|\vec{x}^{\prime}\right\|} \equiv F(t, r) .
$$

The second integral derives from the other nonzero potential $h_{i j}^{(0)}$. Its trace part is obviously the same as $F(t, r)$, and we shall call its traceless part $G(t, r)$,

$$
\begin{aligned}
& \int_{0}^{t} d t^{\prime} \int d^{3} x^{\prime} \theta(\Delta t-\Delta r) {\left[\ln \left(-\mu^{2} \Delta x^{2}\right)-1\right] \times \frac{\widehat{r}^{\prime i} \widehat{r}^{\prime j}}{\left\|\vec{x}^{\prime}\right\|} } \\
& \equiv \frac{1}{2}\left[\delta^{i j}-\widehat{r}^{i} \widehat{r}^{j}\right] F(t, r)-\frac{1}{2}\left[\delta^{i j}-3 \widehat{r}^{i} \widehat{r}^{j}\right] G(t, r), \\
&=\frac{1}{3} \delta^{i j} F(t, r)+\frac{1}{2}\left[3 \widehat{r}^{i} \widehat{r}^{j}-\delta^{i j}\right]\left[G(t, r)-\frac{1}{3} F(t, r)\right] .
\end{aligned}
$$

The integrals are tedious but straightforward and give the following results for $F(t, r)$ and the combination $G(t, r)-\frac{1}{3} F(t, r)$,

$$
\begin{aligned}
& F(t, r)=\frac{4 \pi}{r}\left\{\frac{r^{4}}{6} \ln (2 \mu r)-\frac{25}{72} r^{4}+\frac{11}{18} r^{3} c t-\frac{11}{18} r c^{3} t^{3}+\left[\frac{1}{12}(c t+r)^{4}\right.\right. \\
& \left.\left.-\frac{r}{6}(r+c t)^{3}\right] \ln [\mu(c t+r)]-\left[\frac{1}{12}(c t-r)^{4}+\frac{r}{6}(c t-r)^{3}\right] \ln [\mu(c t-r)]\right\}, \\
& G(t, r)-\frac{F(t, r)}{3}=\frac{4 \pi}{r}\left\{-\frac{r^{4}}{9} \ln (2 \mu r)+\frac{23}{108} r^{4}-\frac{199}{675} r^{3} c t-\frac{13}{135} r c^{3} t^{3}+\frac{2 c^{5} t^{5}}{45 r}\right.
\end{aligned}
$$




$$
\begin{aligned}
& +\left[-\frac{(c t+r)^{6}}{45 r^{2}}+\frac{2}{15} \frac{(c t+r)^{5}}{r}-\frac{5}{18}(c t+r)^{4}+\frac{2}{9} r(c t+r)^{3}\right] \ln [\mu(c t+r)] \\
& \left.+\left[\frac{(c t-r)^{6}}{45 r^{2}}+\frac{2}{15} \frac{(c t-r)^{5}}{r}+\frac{5}{18}(c t-r)^{4}+\frac{2}{9} r(c t-r)^{3}\right] \ln [\mu(c t-r)]\right\} .
\end{aligned}
$$

The next step is acting the two d'Alembertians. This purges all the time dependent terms,

$$
\begin{gathered}
\partial^{4} F(t, r)=\frac{4 \pi}{r} \times 4 \ln (2 \mu r), \\
\partial^{4}\left\{\frac{1}{3} \delta^{i j} F(t, r)+\frac{1}{2}\left[3 \widehat{r}^{i} \widehat{r}^{j}-\delta^{i j}\right]\left[G(t, r)-\frac{1}{3} F(t, r)\right]\right\} \\
=\frac{4 \pi}{r}\left\{\frac{4}{3} \delta^{i j} \ln (2 \mu r)+\left[3 \widehat{r}^{i} \widehat{r}^{j}-\delta^{i j}\right]\left[\frac{4}{3} \ln (2 \mu r)-2\right]\right\} .
\end{gathered}
$$

At this stage the linearized, one loop effective field equations (27) take the form,

$$
\mathcal{D}^{\mu \nu \rho \sigma} h_{\rho \sigma}^{(1)}(t, \vec{x})=\frac{G M}{1280 \pi^{2} c^{2}} \mathrm{D}^{\mu \nu \rho \sigma} f_{\rho \sigma}(\vec{x}),
$$

where the nonzero components of the tensor $f_{\rho \sigma}(\vec{x})$ are,

$$
\begin{aligned}
f_{00}(\vec{x}) & =\frac{4}{r} \ln (2 \mu r) \\
f_{i j}(\vec{x}) & =\delta^{i j} \times \frac{4}{3} \frac{\ln (2 \mu r)}{r}+\left[3 \widehat{r}^{i} \widehat{r}^{j}-\delta^{i j}\right]\left[\frac{4}{3} \frac{\ln (2 \mu r)}{r}-\frac{2}{r}\right] .
\end{aligned}
$$

It remains only to act the operator $\mathrm{D}^{\mu \nu \rho \sigma}$ on $f_{\rho \sigma}(\vec{x})$. The first two derivatives give,

$$
\begin{gathered}
\partial^{\rho} \partial^{\sigma} f_{\rho \sigma}=\frac{4}{r^{3}}, \partial_{j} f_{i j}=\widehat{r}^{i} \frac{[4 \ln (2 \mu r)-4]}{r^{2}}, \\
\partial^{2} f_{00}=-\frac{4}{r^{3}}, \partial^{2} f_{i j}=\delta^{i j} \frac{[8 \ln (2 \mu r)-12]}{r^{3}}-\widehat{r}^{i} \widehat{r}^{j} \frac{[24 \ln (2 \mu r)-32]}{r^{3}}, \\
\partial_{i} \partial_{j} f_{00}=\partial_{k} \partial_{i} f_{j k}, \partial_{i} \partial_{k} f_{j k}=\delta^{i j} \frac{[4 \ln (2 \mu r)-4]}{r^{3}}-\widehat{r}^{i} \widehat{r}^{j} \frac{[12 \ln (2 \mu r)-16]}{r^{3}} .
\end{gathered}
$$

The source term can then be expressed in terms of two more derivatives of the quantities $g_{\mu \nu}(\vec{x}) \equiv \nabla^{2} f_{\mu \nu}(\vec{x})$ and $g(\vec{x}) \equiv \partial_{i} \partial_{j} f_{i j}(\vec{x})$,

$$
\mathcal{D}^{\mu \nu \rho \sigma} h_{\rho \sigma}^{(1)}(t, \vec{x})=\frac{G M}{1280 \pi^{2} c^{2}}\left\{-\eta^{\mu \nu} \nabla^{2} g+\frac{4}{3} \partial^{\mu} \partial^{\nu} g+\frac{1}{3} \nabla^{2} g^{\mu \nu}-\frac{2}{3} \partial_{\rho} \partial^{(\mu} g^{\nu) \rho}\right\} .
$$


The final reduction employs the identities,

$$
\begin{gathered}
\nabla^{2} g=\frac{24}{r^{5}} \quad, \quad \partial_{i} \partial_{j} g=-\frac{12}{r^{5}} \delta^{i j}+\frac{60}{r^{5}} \widehat{r}^{i} \widehat{r}^{j}, \\
\nabla^{2} g_{00}=-\frac{24}{r^{5}} \quad, \quad \nabla^{2} g_{i j}=-\frac{48}{r^{5}} \delta^{i j}+\frac{120}{r^{5}} \widehat{r}^{i} \widehat{r}^{j}, \\
\partial_{k} g_{j k}=-\frac{12}{r^{4}} \widehat{r}^{j} \quad, \quad \partial_{i} \partial_{k} g_{j k}=-\frac{12}{r^{5}} \delta^{i j}+\frac{60}{r^{5}} \widehat{r}^{i} \widehat{r}^{j} .
\end{gathered}
$$

The nontrivial components of the effective field equations are,

$$
\begin{aligned}
& \mathcal{D}^{00 \rho \sigma} h_{\rho \sigma}^{(1)}(t, \vec{x})=\frac{G M}{80 \pi^{2} c^{2}} \times \frac{1}{r^{5}} \\
& \mathcal{D}^{i j \rho \sigma} h_{\rho \sigma}^{(1)}(t, \vec{x})=\frac{G M}{80 \pi^{2} c^{2}} \times\left\{-\frac{3 \delta^{i j}}{r^{5}}+\frac{5 \hat{r}^{i} \widehat{r}^{j}}{r^{5}}\right\} .
\end{aligned}
$$

\subsection{The One Loop Potentials}

We wish to express the one loop potentials in Schwarzschild coordinates so their nonzero components take the form,

$$
h_{00}^{(1)}(\vec{x})=a(r) \quad, \quad h_{i j}^{(1)}(\vec{x})=\widehat{r}^{i} \widehat{r}^{j} b(r) .
$$

Acting the Lichnerowitz operator (3) on these gives,

$$
\begin{aligned}
& \mathcal{D}^{00 \rho \sigma} h_{\rho \sigma}^{(1)}=\frac{b^{\prime}}{r}+\frac{b}{r^{2}}, \\
& \mathcal{D}^{i j \rho \sigma} h_{\rho \sigma}^{(1)}=\delta^{i j}\left[-\frac{a^{\prime \prime}}{2}-\frac{a^{\prime}}{2 r}-\frac{b^{\prime}}{2 r}\right]+\widehat{r}^{i} \widehat{r}^{j}\left[\frac{a^{\prime \prime}}{2}-\frac{a^{\prime}}{2 r}+\frac{b^{\prime}}{2 r}-\frac{b}{r^{2}}\right] .
\end{aligned}
$$

Comparing (51) with (48) implies,

$$
b(r)=\frac{G M}{160 \pi^{2} c^{2}} \times-\frac{1}{r^{3}} .
$$

Substituting this in (52) and comparing with (49) implies,

$$
a(r)=\frac{G M}{160 \pi^{2} c^{2}} \times \frac{1}{r^{3}} .
$$

Combining the classical and quantum corrections gives the following total results for the potentials,

$$
\begin{aligned}
h_{00}(\vec{x}) & =\frac{2 G M}{c^{2} r}\left\{1+\frac{\hbar G}{20 \pi c^{3} r^{2}}+O\left(\frac{\kappa^{4}}{r^{4}}\right)\right\}, \\
h_{i j}(\vec{x}) & =\frac{2 G M}{c^{2} r}\left\{1-\frac{\hbar G}{20 \pi c^{3} r^{2}}+O\left(\frac{\kappa^{4}}{r^{4}}\right)\right\} \widehat{r}^{i} \widehat{r}^{j} .
\end{aligned}
$$




\section{Discussion}

We have used the Schwinger-Keldysh formalism to derive manifestly causal and real, linearized effective field equations (27) for $h_{\mu \nu}(t, \vec{x})$ which include the one loop quantum effects of a massless, minimally coupled scalar. We used this equation to solve for the quantum corrections to the potentials (5556) associated with a stationary point mass. Similar results have been obtained for conformally coupled scalars, fermions and vectors [5, 6]. The massless, minimally coupled scalar result does not seem to have been previously computed, but the real power of our analysis is the field equation (27), which could be used even for a time dependent source. Our results (55+56) will also serve as an important correspondence limit for an on-going computation of the quantum corrected potentials on a locally de Sitter background.

An important caveat to this analysis is that the full linearized effective field equations must include quantum corrections to the stress-energy of the source as well as corrections to the graviton kinetic operator. For our problem, the scalar makes no contribution to the stress-energy of the point mass which was our source. However, if we had considered quantum gravitons it would have been necessary to correct the stress-tensor, and some account would also have to be taken of the fact that the potentials would be measured by coupling through a corrected vertex. These complications are among the reasons why it required so much more work to derive reliable results for gravitons [1, 7, 8, 9, 10, 11, 12].

Finally, it is worth noting that the massless, minimally coupled scalar stands in isolation to its conformally coupled cousin. The self-energies of both scalars take the same form,

$$
\begin{gathered}
\text { Minimally Coupled } \Longrightarrow\left[{ }^{\mu \nu} \Sigma_{ \pm \pm}^{\rho \sigma}\right]=( \pm)( \pm) \frac{i \kappa^{2} \mathrm{D}^{\mu \nu \rho \sigma} \partial^{2}}{5120 \pi^{4}}\left[\frac{\ln \left(\mu^{2} \Delta x_{ \pm \pm}^{2}\right)}{\Delta x_{ \pm \pm}^{2}}\right],(57) \\
\text { Conformally Coupled } \Longrightarrow\left[{ }^{\mu \nu} \widetilde{\Sigma}_{ \pm \pm}^{\rho \sigma}\right]=( \pm)( \pm) \frac{i \kappa^{2} \widetilde{\mathrm{D}}^{\mu \nu \rho \sigma} \partial^{2}}{5120 \pi^{4}}\left[\frac{\ln \left(\mu^{2} \Delta x_{ \pm \pm}^{2}\right)}{\Delta x_{ \pm \pm}^{2}}\right] .(58)
\end{gathered}
$$

However, the 4 th-order operator $\mathrm{D}^{\mu \nu \rho \sigma}$ has a nonzero trace whereas $\widetilde{\mathrm{D}}^{\mu \nu \rho \sigma}$ is traceless,

$$
\begin{aligned}
\mathrm{D}^{\mu \nu \rho \sigma} & =\Pi^{\mu \nu} \Pi^{\rho \sigma}+\frac{1}{3} \Pi^{\mu(\rho} \Pi^{\sigma) \nu}, \\
\widetilde{\mathrm{D}}^{\mu \nu \rho \sigma} & =-\frac{1}{9} \Pi^{\mu \nu} \Pi^{\rho \sigma}+\frac{1}{3} \Pi^{\mu(\rho} \Pi^{\sigma) \nu},
\end{aligned}
$$


where $\Pi^{\mu \nu} \equiv \eta^{\mu \nu} \partial^{2}-\partial^{\mu} \partial^{\nu}$. The graviton self-energy from a massless, 2component fermion is three times that of the massless, conformally coupled scalar (58), and the result for a massless vector is 12 times (58), so the massless, minimally coupled scalar is the exceptional case. This distinction becomes even greater in de Sitter background on which massless, minimally coupled scalars experience explosive particle production whereas the various massless, conformally invariant particles do not. (For a simple discussion, see section III of [31].)

\section{Acknowledgements}

It is a pleasure to acknowledge correspondence on this subject with S. Deser and J. F. Donoghue. This work was partially supported by NSF grants PHY-0653085 and PHY-0855021 and by the Institute for Fundamental Theory at the University of Florida.

\section{References}

[1] A. F. Radkowski, Ann. Phys. 56 (1970) 319.

[2] D. M. Capper, M. J. Duff and L. Halperin, Phys. Rev. D10 (1974) 461.

[3] D. M. Capper and M. J. Duff, Nucl. Phys. B84 (1974) 147.

[4] D. M. Capper, Nuovo Cimento A25 (1975) 29.

[5] M. J. Duff, Phys. Rev. D9 (1974) 1837.

[6] M. J. Duff and J. T. Liu, Phys. Rev. Lett. 85 (2000) 2052, hep-th/0003237.

[7] J. F. Donoghue, Phys. Rev. Lett. 72 (1994) 2996, gr-qc/9310024; Phys. Rev. D50 (1994) 3874, gr-qc/9405057.

[8] I. J. Muzinich and S. Kokos, Phys. Rev. D52 (1995) 3472, hep-th/9501083.

[9] H. Hamber and S. Liu, Phys. Lett. B357 (1995) 51, hep-th/9505182.

[10] A. Akhundov, S. Belucci and A. Shiekh, Phys. Lett. B395 (1997) 16, gr-qc/9611018. 
[11] I. B. Khriplovich and G. G. Kirilin, J. Exp. Theor. Phys. 95 (2002) 981, gr-qc/0207118 J. Exp. Theor. Phys. 98 (2004) 1063, gr-qc/0402018.

[12] N. E. J. Bjerrum-Bohr, J. F. Donoghue and B. R. Holstein, Phys. Rev. D67 (2003) 084033, Erratum-ibid. D71 (2005) 069903, hep-th/0211072.

[13] J. Schwinger, Phys. Rev. 82 (1951) 914; Phys. Rev. 91 (1953) 713.

[14] E. Witten, "Quantum Gravity in de Sitter Spcae," hep-th/0106109.

[15] A. Strominger, JHEP 0110 (2001) 034, hep-th/0106113.

[16] J. Schwinger, J. Math. Phys. 2 (1961) 407; K. T. Mahanthappa, Phys. Rev. 126 (1962) 329; P. M. Bakshi and K. T. Mahanthappa, J. Math. Phys. 4 (1963) 1; J. Math. Phys. 4 (1963) 12; L. V. Keldysh, Sov. Phys. JETP 20 (1965) 1018.

[17] L. H. Ford and R. P. Woodard, Class. Quant. Grav. 22 (2005) 1637, gr-qc/0411003.

[18] E. O. Kahya, V. K. Onemli and R. P. Woodard, Phys. Rev. D81 (2010) 023508, arXiv:0904.4811.

[19] K. C. Chou, Z. B. Su, B. L. Hao and L. Yu, Phys. Rept. 118 (1985) 1; R. D. Jordan, Phys. Rev. D33 (1986) 444; E. Calzetta and B. L. Hu, Phys. Rev. D35 (1987) 495.

[20] N. C. Tsamis and R. P. Woodard, Phys. Rev. D54 (1996) 2621, hep-ph/9602317.

[21] T. Prokopec, O. Tornkvist and R. P. Woodard, Phys. Rev. Lett. 89 (2002) 101301, astro-ph/0205331; Annals Phys. 303 (2003) 251, gr-qc/0205130.

[22] T. Prokopec and R. P. Woodard, Annals Phys. 312 (2004) 1, gr-qc/0310056.

[23] E. O. Kahya and R. P. Woodard, Phys. Rev. D72 (2005) 104001, gr-qc/0508015 Phys. Rev. D74 (2006) 084012, gr-qc/0608049.

[24] T. Prokopec and R. P. Woodard, JHEP 0310 (2003) 059, astro-ph/0309593; B. Garbrecht and T. Prokopec, Phys. Rev. D73 (2006) 064036, gr-qc/0602011. 
[25] L. D. Duffy and R. P. Woodard, Phys. Rev. D72 (2005) 024023, hep-ph/0505156.

[26] T. Brunier, V. K. Onemli and R. P. Woodard, Class. Quant. Grav. 22 (2005) 59, gr-qc/0408080; E. O. Kahya and V. K. Onemli, Phys. Rev. D76 (2007) 043512, gr-qc/0612026.

[27] S. P. Miao and R. P. Woodard, Class. Quant. Grav. 23 (2006) 1721, gr-qc/0511140 Phys. Rev. D74 (2006) 044019, gr-qc/0602110.

[28] E. O. Kahya and R. P. Woodard, Phys. Rev. D76 (2007) 124005, arXiv:0709.0536DA; Phys. Rev. D77 (2008) 084012, arXiv:0710.5282.

[29] A. Campos and E. Verdaguer, Phys. Rev. D49 (1994) 1816, gr-qc/9307027; R. Martin and E. Verdaguer, Phys. Rev. D61 (2000) 124024, gr-qc/0001098.

[30] G. 't Hooft and M. Veltman, Ann. Inst. Henri Poincaré, XX, 69 (1974).

[31] T. Prokopec and R. P. Woodard, Am. J. Phys. 72 (2004) 60, astro-ph/0303358. 